\title{
LA VOCE DEL NARRATORE \\ NEL POEMA CAVALLERESCO RINASCIMENTALE \\ ITALIANO
}

\section{ENRICO MUSACCHIO}

\begin{abstract}
Riassunto: Il discorso sul ruolo della 'voce' del narratore nel racconto letterario è al centro della riflessione narratologica contemporanea. Ma già nel Cinquecento, e in particolare a proposito dei romanzi cavallereschi, sempre popolari ma ormai sospettati di infrangere le norme della poetica aristotelica, il problema dell'estensione e delle forme letterariamente accettabili per l'intervento del 'narratore', è vivacemente dibattuto. Alla discussione partecipano i letterati che ambiscono di inventare una nuova poetica del poema eroico e i teorici della letteratura più o meno impregnati dell'aristotelismo dominante.
\end{abstract}

Nel suo trattato del 1554 su I Romanzi, Giovambattista Pigna afferma che Ariosto, nell' Orlando Furioso, ha adeguatamente ed equamente impiegato ("l'una et l'altra ha la sua ragionevole parte") i due modi in cui si può eseguire un racconto, e cioè "nel narrare o nell'inducere altri a ragionamento" (80).

La menzione di questa duplice possibilità narrativa, raccontare con la propria voce o far parlare i personaggi, fa ovviamente riferimento alla Poetica di Aristotele, il quale distingue, nel capitolo III, tra la forma narrativa in cui l'autore racconta in persona propria quello che viene detto dai vari personaggi, e la forma drammatica in cui l'autore mette nella bocca dei personaggi i loro propri discorsi: "il poeta può imitare in due modi diversi e cioè, o in forma narrativa ... o in forma drammatica" (1448a 20)1. Alessandro Piccolomini, pochi anni dopo, nel 1575, così tradurrà il passo

${ }^{1}$ Il testo utilizzato è in Aristotle, Poetics, a cura di Lucas. La traduzione è quella di Galvano Della Volpe (Della Volpe, Poetica del Cinquecento, 60). Rostagni (Aristotele, Poetica, a cura di Rostagni, nota a p. 14) così parafrasa il passo aristotelico: "è possibile imitare... in modo diverso, ossia, 1.o) in forma narrativa ... raccontando ... conservandosi l'autore ... in persona propria ... e non cambiando; 2.o) in forma drammatica ... il poeta affida l'imitazione uscendo dalla propria persona e trasformandosi nelle persone ... ad agire ed operare direttamente". 
aristotelico: "per via di narratione et di raccontamento et questo o ponendo se stesso alle volte il poeta in persona d'altri con diventar in un certo modo la cosa imitata come fa Homero over conservando sempre la propria persona non mutata mai"2.

A sua volta questa distinzione aristotelica trae origine da Platone che, nel III Libro della Repubblica, la chiarisce con uno splendido esempio preso da Omero. La questione trattata da Platone è quella della "mimesi", e il problema della distanza più o meno grande che vi è tra il discorso umano e la 'realtà' a cui il discorso si riferisce e che intende in qualche modo riprodurre. In questo contesto, Platone distingue due livelli di imitazione, quando il "poeta stesso" racconta, e quando invece il poeta inventa personaggi che parlano; il primo è una "narrazione semplice", il secondo una "narrazione mimetica". Nel corso del dialogo Socrate distingue tre modi di racconto (DIËGESIS): "con una narrazione semplice (APLÈ DIEGÈSEI)", "con una narrazione mimetica (DIÀ MIMĖSEOS)", "con una narrazione mista (DI'AMFOTĖRON)". La contrapposizione proposta da Socrate al suo interlocutore sarà tra un Omero che parla in nome proprio (AUTÒS O POIETES) e un Omero che si sforza di farci credere che non è Omero che parla ma il vecchio sacerdote Crise (ME OMERON DOKEIN EINAI TON LEGONTA ALLÀ TON IEREA)3. Questo secondo è quello che viene propriamente definito, e particolarmente condannato, come "imitazione". Per spiegare ciò che intende dire, Platone offre una versione modificata del primo episodio narrato nell' Iliade, la scena in cui Crise chiede ad Agamennone di liberare la figlia. Omero introduce il sacerdote che si indirizza ai Greci ("pregava tutti gli Achei ma sopratutto i due Atridi") e riproduce poi in discorso diretto le parole stesse dell'invocazione di Crise: "Atridi, e voi tutti, Achei schinieri robusti, a voi diano gli Dei ... d'abbattere la città di Priamo, di ben tornare in patria, e voi liberate la mia creatura" (nella traduzione di Rosa Calzecchi Onesti). Riscrivendo questo passo nella Repubblica, Platone fornisce una versione in discorso indiretto, un racconto che definirà come "narrazione semplice" invece della "narrazione per via mimetica" fornita da Omero: "Venuto il sacerdote, pregò che a loro gli dei concedessero di prendere Troia e di salvarsi, ed essi a lui riconsegnassero la figlia, ricevendone il riscatto" 4 . Nel dialogo platonico, al centro della discussione vi è la questione della accettabilità o meno, nella città ideale, di quella 'realtà' illusoria che è l'essenza stessa della poesia, e

2 Piccolomini, Annotationi, 52.

3 Platone, La Repubblica, Libro III (392d), (393a e 393b).

${ }^{4}$ Platone, La Repubblica, Libro III (393e). 
dunque del suo massimo esponente, Omero. Naturalmente alla base del discorso vi è lo specifico nucleo filosofico del platonismo che contrappone al 'vero' le versioni, più o meno distanti, che la 'imitazione' umana ne può dare. Ed è appunto in questo contesto filosofico che viene posta la distinzione letteraria tra 'racconto puro e semplice' (racconto 'diegetico') e 'racconto drammatizzato' (racconto 'mimetico'), il quale ultimo, attraverso il gioco dell'attore o anche semplicemente del narratore che racconta in discorso diretto, aggiunge un ulteriore elemento di illusorietà. Il fatto di chiarire il carattere più palesemente mimetico della letteratura drammatica e anche della narrativa che prende in prestito il modo drammatico, serve dunque a mostrare il divario tra, da un canto, la narrazione di un discorso e, dall'altro, la riproduzione mimetica di quello stesso discorso per bocca di un attore o di un personaggio del racconto. In seguito tuttavia, nel Libro $X$ della Repubblica, tutta l'opera del poeta, sia quella nel modo drammatico che quella nel modo narrativo, verrà denunciata come una copia imperfetta di una realtà che a sua volta è copia (imitazione) di una realtà superiore, il mondo delle idee: nulla di meglio dunque che una copia di una copias.

Della discussione sulla poesia di Platone, e della sua identificazione, così singolarmente piena di acume, dei due modi del racconto, nel capitolo III della sua Poetica Aristotele non ha solo ritenuto la terminologia, ma anche - come si è visto - la distinzione stess $a^{6}$. Tuttavia non ha fatto sua la condanna metafisica dell'arte come semplice illusione. Per Aristotele

5 "La mimesi" non è altro che "un gioco e non una cosa seria". Platone, La Repubblica, Libro X (602b); i poeti "fanno pure parvenze e non enti reali" (599a); "l'arte mimetica è lontana della verità" (603a); "mettiamo a cominciare da Omero che tutti i poeti siano imitatori di parvenze" (600e); i poeti in effetti imitano delle imitazioni della vera realtà del mondo delle 'idee', e pertanto creano opere "che distano di tre distanze dalla vera realtà" (599a).

${ }^{6}$ Non solo i termini impiegati da Aristotele, come 'mimesi' e 'diegesi', sono quelli stessi di Platone, ma anche certe espressioni come 'il poeta stesso' per dire 'il poeta in persona propria', 'divenire un altro' per 'esprimere in discorso diretto le parole di un personaggio'. Il passo del Capitolo III della Poetica aristotelica è molto complesso (anche sintatticamente) e i commentatori hanno fornito diverse spiegazioni. Il problema delle somiglianze e delle differenze tra Platone e Aristotele su tale soggetto è reso più complicato dal fatto che, mentre è chiaro che Platone (si veda Repubblica, 394c) traccia una tripartizione tra una forma puramente imitativa, la 'drammatizzazione' (esemplificata dalla tragedia e dalla commedia), una forma di solo racconto (esemplificata dal ditirambo), e una forma mista (esemplificata dalla poesia epica), invece, per quanto riguarda Aristotele, non è chiaro se i modi siano due o tre. Si veda l'analisi delle varie interpretazioni in Else, Aristotle's Poetics: The Argument, 90-101. 
infatti l'arte è per propria natura 'mimesi'; e, in poesia, sia la forma 'narrativa pura e semplice' sia la forma 'drammatica' partecipano a egual titolo di questa natura imitativa che ne è l'essenza stessa. Nel capitolo III della Poetica i due modi del narrare sono dunque posti sullo stesso piano.

E tuttavia, nel capitolo XXIV, una sorprendente riflessione verrà aggiunta da Aristotele: "Omero per infinite altre ragioni è degno di lode, ma in modo specialissimo per questa, che egli è l'unico dei poeti epici il quale non ignori qual parte il poeta deve assumere nel poema in propria persona. Il poeta epico deve parlare in persona propria il meno che è possibile; quando fa cotesto, egli non è imitatore (nello stretto senso della parola). Gli altri poeti entrano sempre in campo con la propria persona; poco o raro si immedesimano in coloro che vogliono rappresentare. Omero invece, dopo poche parole come di presentazione, subito introduce o un uomo o una donna o qualche altro carattere" (1460a5)7. Giudizio sorprendente perché Aristotele, che aveva definito la narrativa tanto quanto la drammatica come forme ugualmente 'mimetiche', sembra ora stabilire una scala di valore mimetico che pone alla sommità il dramma, mentre l'epica sarebbe meno mimetica (e dunque meno poetica), sebbene poi fra i poeti epici Omero goda di una netta superiorità perché è quello che più si avvicina alla forma drammatica in quanto, nel genere misto (che è quello

7 Nella versione di Della Volpe (153). Così traduce Alessandro Piccolomini nelle sue Annotationi al testo aristotelico: "egli fra gli altri poeti sa molto bene ciò che convenga fare a un tal poeta. Et questo è che convien ch'il poeta pochissimo dica nella propria persona sua, non essendo così vero imitatote mentre ch'egli fa quello", 384 .

${ }^{8}$ Varie sono le interpretazioni dei moderni commentatori per spiegare la divergenza del punto di vista sulla mimesi poetica che appaiono nei due passi aristotelici, da Else (Aristotle's Poetics: The Argument, 620-621) per il quale Aristotele, nel suo entusiasmo per Omero, fa questa affermazione 'paradossale' e in contrasto con la dichiarazione precedente (mentre per Rostagni, Aristotele a questo punto ha semplicemente dimenticato altre forme poetiche "onde dal campo della poesia viene ad essere esclusa, particolarmente, la vera e propria lirica", 149) a Halliwell (Aristotle's Poetics. in particolare il cap. IV, 109-137) che in un'analisi lucidissima tenta di districare i vari sensi della MIMESIS aristotelica fra "imitation" e "enactment". Si noti che, proprio per questa preponderanza della 'narrazione mimetica' sulla 'narrazione semplice' tanto ammirata da Aristotele, Platone aveva al contrario formulato grandi riserve sul ruolo di Omero come modello quando, in una narrazione che comprendesse (come in Omero) i due elementi, invitava a sforzarsi di introdurre solamente "una piccola parte di mimesi in un lungo discorso" (La Repubblica, Libro III (396e). 
epico), egli riduce al minimo la componente narrativa ${ }^{8}$.

È dunque chiaro che Pigna, nei Romanzi, al momento di discutere l'arte di Ariosto e di giustificarne le scelte estetiche, tiene conto non solo di quanto Aristotele dice nel capitolo III della Poetica, ma anche del giudizio più problematico contenuto nel capitolo XXIV. Infatti, come si è visto, Pigna inizia dicendo che Ariosto è stato capace di usare in modo soddisfacente sia la 'forma narrativa' sia la 'forma drammatica'; ma, con ovvio riferimento al giudizio ulteriore pronunziato da Aristotele nei riguardi di Omero nel capitolo XXIV della Poetica, aggiunge: "quantunque il narrare da sé tanta imitatione non habbia, nondimeno perché alla gravità ciò molto riguarda, che è una essential parte dell'Epico, senza che per lo narrare da sé è dal Tragico differente, è stato necessario che anchora a ciò massimamente egli si dia" (82). Il "narrare da sé", ammette Pigna riprendendo il giudizio di Aristotele, non è così 'imitativo' (e dunque non corrisponde a quella che è l'esigenza essenziale della poesia) quanto "l'inducere altri a ragionamento", ma è inevitabile nel caso della poesia Epica (e dunque nel caso dell' Orlando Furioso) e non può pertanto essere imputato a difetto dell'opera.

Ma per ben intendere le parole di Pigna è necessario guardare, al di là dei riferimenti ad Aristotele, al contesto delle discussioni letterarie dell'epoca, e in particolare alle polemiche intorno all' Orlando Furioso, di cui il trattato I Romanzi assume la difesa.

Tutta questa seconda parte del trattato di Pigna consiste infatti in una risposta ai detrattori dell'Ariosto. Primo fra questi è Trissino, identificato non per nome ma mediante una citazione dal suo poema L'Italia liberata da Gotthi, in cui il Furioso è menzionato come il poema "che piace al volgo" ("Benché dicano alcuni che il Furioso è per lo volgo, essi nientedimeno auttorità alcuna intorno a ciò haver non possono, essendo che niuno è che loro creda, ritrovandogli di gusto non pur guasto ma corrotto del tutto, percioché s'essi hanno il cattivo d'Homero per ottimo, et per pessimo il buono del medesimo da Vergilio imitato et da tutti i più savi infinitamente lodato") ${ }^{9}$. Non solo dunque Trissino è identificato con la parola "volgo", che riprende una citazione dall' Italia liberata (Libro XXIV), ma il giudizio viene inserito nel contesto della controversia pro Omero o pro Virgilio. Il verso di Trissino aveva fatto scorrere molto inchiostro da parte di letterati (sopratutto quelli ferraresi) indignati per il suo commento sprezzante sulla popolarità dell'Orlando Furioso, indice della sua mancanza di qualità

9Pigna, I Romanzi, 68.

${ }^{10}$ Si veda a tal proposito Giraldi Cinzio, Discorso dei romanzi, 66, e la nota 70. 
estetiche, della sua volgarità insomma ${ }^{10}$. Nella fattispecie poi lo stesso Pigna, nelle pagine che seguono, darà come maggior prova del valore del poema di Ariosto proprio la sua popolarità. Verso la fine del secolo, nel 1589, Giuseppe Malatesta, nel Dialogo della Nuova Poesia, farà dibattere ai suoi personaggi la questione se la popolarità di un'opera sia indice di eccellenza o del suo contrario. Una delle opinioni profferte è che "il Furioso diletti in gran maniera agli huomini rozzi et volgari" mentre invece "il Poeta per meritare questo nome deve recar dilettatione solo agli huomini dotti et eruditi" 11 . I detrattori del Furioso a cui Pigna si riferisce vengono definiti come "abbaiatori ... che se ben per rabbia dall'invidia incitata di mordere l'Ariosto s'affaticano, cercano di far piaga di poca valuta, percioché ella da se stessa si sanerà, dovendo essere la fama vera medicina di tal morso" (68). Chi siano questi "abbaiatori", questi "morditori”, come li definirà nelle pagine che seguono ("Se per aventura troppo caldamente contra i morditori di lui mosso mi sono") ${ }^{12}$, non viene precisato da Pigna, ma e' chiaro che si tratta delle stesse persone a cui aveva fatto riferimento in una Lettera indirizzata a Giraldi Cinzio e datata "25 luglio 1548, di Lucca" ("Se coloro ... ch'oggidì stanno su il mordere gli altrui componimenti, si dessero a scrivere et a mandare in luce le lor composizzioni, forse che in questo mio viaggio di Toscana non avrei trovato tanti morditori dell'Ariosto") 13 .

In effetti poi, in quella Lettera del 1548, Pigna non aveva menzionato, fra le pecche in cui (secondo i critici toscani) Ariosto sarebbe incorso, lo specifico problema del preponderare del 'narrativo' sul 'drammatico'. Ma la menzione, nei Romanzi del 1554, di questa possibile critica ("quantunque il narrare da sé tanta imitatione non habbia"), per il fatto di venir strettamente collegata ai "morditori", sembrerebbe suggerire che fra i tipi di "piaga di poca valuta" inferti da coloro alla rinomanza del gran poeta ferrarese, c'era stato anche questo, e cioè che egli troppo avesse usato il "narrare da sé". Non era apertamente menzionato fra i difetti (per i critici toscani) dell'Ariosto nella Lettera di Pigna del 1548; e non se ne era discusso nella lunga lettera di risposta, sempre datata 1548, in cui Giraldi elabora la sua prima versione del Discorso dei romanzilit. Dal momento che

${ }^{11}$ Malatesta, Della nuova poesia, 154.

12 Pigna, I Romanzi, 70.

${ }^{13}$ Lettera di G. Pigna del XXV luglio 1548 in Giraldi Cinzio, Discorso dei romanzi, 224.

${ }^{14}$ Lettera di G. Giraldi del I agosto 1548 in Giraldi Cinzio, Discorso dei romanzi, 224-229. 
esiste il dubbio che le circostanze di questo scambio di lettere siano state inventate dai due partecipanti, e che invece essi avessero convenuto su quali delle critiche ad Ariosto andassero menzionate e quali no, e quali in particolare dovessero essere oggetto delle repliche di Giraldi15, è impossibile concludere sulle ragioni per le quali questa critica, che riaffiorerà qualche anno dopo nei Romanzi del Pigna, non venga invece menzionata nel 1548. Rimane insomma senza risposta il nostro dubbio se, nel 1548, Pigna e Giraldi non la abbiano menzionata perché la ritenessero senza fondamento, o perché al contrario la ritenessero valida e non sapessero come difenderne Ariosto.

Come si è visto Pigna ne parlerà in seguito nei Romanzi del 1554. La questione non sarà invece affrontata in quanto tale nel Discorso dei romanzi di Giraldi, pure del 1554, sebbene se ne possa trovare effettivamente una traccia nel Discorso dei romanzi, ma soltanto in riferimento ad un altro distinto problema. Parlando della divisione dei Romanzi in 'canti', Giraldi cerca di prendere le distanze da una interpretazione dell'origine di quest'uso che egli teme possa svilire il programma estetico dei due autori degli Orlandi: "Né questo nome di canto, dato a tali poemi, è nato appresso noi perché per le piazze et per gli luochi publici si cantassero per su le panche queste composizioni, a guisa che fanno oggidì costoro che con la lira in braccio cantano le lor fole et così si guadagnano il pane, ma da più alto et da più onorato principio è egli nato questo nome" (37). E Giraldi, nel tentativo di trovare un'origine più nobile, afferma la differenza strutturale tra Romanzi e poemi epici classici: "i nostri poeti, i quali hanno così le loro composizioni divise, voltano spesse volte il loro parlare a quelle persone dinanzi alle quali fingono di cantare. Cosa che non sarebbe convenevole a' poeti greci et latini et volgari che componessero nel modo nel quale ha composto Vergilio et Omero, perché essi sono narratori da sé et non hanno questo rispetto se non in quanto introducono alcuno che narri i suoi errori overo i suoi fatti o quelli degli altri, come si vede in Omero di Ulisse, et di Enea in Vergilio. Et questo voglio che per ora basti quanto al nome dei romanzi, all'origine loro et alla divisione dei lor poemi in canti" (38). Vengono contrastati dunque i "narratori da sé" e i narratori che "introducono alcuno che narri”, ma senza discutere la distinzione stessa. Essa è presentata semplicemente come nata da una prassi poetica diversa. Giraldi insomma, sebbene sia conscio dei dati del problema, come rivela l'impiego dei termini rilevanti (i "narratori da sé", e quelli invece che "introducono qualcuno che narri"), non sembra aver colto (come invece Pigna nella sua

${ }^{15}$ Musacchio, "Il 'principio, mezzo e fine' del poema". 
pur breve menzione) la specificità della questione aperta dalla distinzione dei due modi di eseguire un racconto e del problema della preferenza assegnata da Aristotele al modo 'drammatico'.

A quali fonti potevano attingere Pigna e Giraldi nell'elucidazione della Poetica aristotelica? La versione latina della Poetica, eseguita da Guglielmo di Moerbeke, è in effetti sufficientemente esplicita. I nostri due passi sono così tradotti in tale versione: quello dal capitolo III dice: "Et enim in eisdem et eadem imitari est aliquando quidem enuntiantem aut alterum aliquid gerentem sicut Homerus facit, aut ut eundem et non transmutantem, aut omnes ut actitantes et operantes imitantes"; quello dal capitolo XXIV dice: "Homerus autem alias in multis dignus laudari, et etiam quia solus poetarum non ignorat quod oportet poetizare ipsum. Ipsum enim utique poetam paucissima prologizare. Non enim est secundum hec imitator. Alii quidem igitur ipsi quidem per totum agonizant, imitantur autem pauca et raro; hic autem pauca prohemizans mox inducit virum aut mulierem aut alium aliquem"16. Tuttavia questa traduzione di Guglielmo di Moerbeke del testo aristotelico è rimasta completamente sconosciuta nel Rinascimento ${ }^{17}$. E per quel che riguarda la versione commentata di Averroé, che fino alla traduzione di Valla, era l'unico modo per conoscere il pensiero aristotelico in materia di poetica, essa in questo caso è totalmente inutilizzabile ("E conviene che ciò che il poeta dice a proprio nome sia poco correlato al discorso rappresentativo come fa Omero: egli infatti compone un piccolo esordio e poi va verso ciò che vuole rappresentare senza addurre in ciò nulla che sia inadatto ma ciò che è abituale") ${ }^{18}$. Averroé produce uno stravolgimento totale del testo della Poetica che per ragioni culturali gli è incomprensibile.

Nel 1554 Pigna e Giraldi, peraltro, avevano ormai a disposizione due dei grandi commentari rinascimentali della Poetica aristotelica, quello di Robortello e quello di Lombardi-Maggi (in particolare Maggi era stato da loro molto apprezzato come professore e come collega a Ferrara); e si potrebbe immaginare che sia stato per merito di questi commentari che $\mathrm{i}$ due trattatisti del 'Romanzo' abbiano potuto prender coscienza del problema. In realtà né l'una né l'altra di queste due opere ha posto un'attenzione specifica alla questione.

Commentando il difficile passo del capitolo III della Poetica aristoteli-

\footnotetext{
${ }^{16}$ Dalla traduzione di William de Moerbeck (in Aristotele, De arte poetica, 5 e 31 ).

17 "That translation was completely unknown during the Renaissance", Weinberg, A History of Literary Criticism in the Italian Renaissance, 1:352.

18 Averroé, Commento al 'Perì Poietikês', 120.
} 
ca, Robortello, nelle sue Explicationes del 1548, dichiara di ritenere erronea l'interpretazione dei due traduttori latini della Poetica, Valla e Pazzi. Secondo Robortello i due modi narrativi vengono riferiti da Aristotele non ai poeti dei poemi epici ma ai personaggi, i quali possono fare $\mathrm{i}$ loro racconti sia con narrazioni che con drammatizzazioni ("Hic est verus, ut opinor, Aristotelis sensus in illis verbis ... ut illam dictionem ... referas ad personam quam imitatur poeta, not ad poetam sicuti retulit Paccius") ${ }^{19}$. E Robortello cita parallelamente l'esempio in Omero del racconto di Ulisse ad Alcinoo e l'esempio in Virgilio del racconto di Enea a Didone ("Licet imitari aliquem tamquam nunciantem et alterius subinde personam suscipientem, id quod facit Homerus. Nam Homerus imitatur Ulyssem nunciantem et narrantem Alcinoo regi omnia ... Idem facit Vergilius apud nos Libro Secundo Aeneidos"20. Si tratta dunque di un espediente da impiegare per variare i discorsi che i diversi personaggi faranno nel corso degli eventi raccontati, non di un'alternativa stilistica che si offre al poeta al momento di comporre il suo racconto. Sparisce dunque nell'interpretazione di Robortello il contrasto tra due modi di comporre un poema epico, e di conseguenza anche una possibile polemica contro gli autori di Romanzi eccessivamente inclini (come appunto è probabile che apparisse Ariosto ai suoi "morditori") a introdurre la narrazione in persona propria.

Lombardi e Maggi, a differenza di Robortello, affermano invece, nelle loro Communes Explanationes del 1550, che la narrazione in persona propria e la drammatizzazione erano da Aristotele assegnate al poeta: "Poetae ... diversis tamen modis imitari queunt ... interdum ipsos loqui semper nec alienam personam induere, ut in dythirambis ... nunnumquam alios perpetuo loquentes inducere, ut in trageoediis atque comoediis efficiunt, quandoque vero paucis admodum a se prolatis poetae verbis loquentem aliquem introducunt"21. Maggi poi, nelle sue proprie Annotationes dello stesso volume, riportava l'intero passo platonico della Repubblica facendo osservare che Aristotele aveva migliorato l'idea di Platone ("deprehendi possit quam docte placitis Platonis sui praeceptoris Aristoteles utens in melius ea reformet"), per il fatto di dichiarare che i due modi del racconto, e non la sola drammatizzazione, sono mimetici ("Aristoteles ... nullam

${ }^{19}$ Robortello, In librum Aristotelis de arte poetica explicationes, 24.

${ }^{20}$ Si noti che Pigna fa suoi i due esempi impiegati da Robortello.

${ }^{21}$ Maggi, In AristotelislLibrum de poetica communes rxplanationes, 24. È il frutto del comune lavoro interpretativo di Maggi e di Lombardi (deceduto probabilmente nel 1541: si veda Weinberg, A History of Literary Criticism in the Italian Renaissance, 1:373). 
esse absque imitatione Poesim operis huius initio declaravit")22.

Né il commentario di Robortello né quello di Lombardi e Maggi, tuttavia, si soffermano ad esaminare e tentare di spiegare quel passo del capitolo XXIV della Poetica dove, come abbiamo visto, Aristotele, distanziandosi radicalmente dalla condanna platonica, nella sua lode dell'abilità di Omero di "parlare in persona propria il meno che è possibile", dà esplicitamente la palma al modo drammatico in quanto più mimetico rispetto al modo narrativo semplice.

Quando dunque, nei Romanzi, Pigna, difendendo l'estetica ariostesca, aveva menzionato i due modi del narrare così come sono contenuti nel capitolo III della Poetica aristotelica, ma anche il giudizio contenuto nel capitolo XXIV secondo cui il modo 'drammatico' è superiore perché più imitativo, non poteva essersi avvalso delle analisi dei due grandi commentatori, i quali sono silenziosi al riguardo. E questo sembra confermare il fatto che la critica formulata contro Ariosto (e cioè che nel Furioso il poeta/narratore interviene troppo) sia arrivata a Pigna durante il suo viaggio in Toscana; dunque in ambienti culturalmente vicini a Trissino (indicato appunto da Pigna come il capofila dei 'morditori' dell'Ariosto) il quale aveva, come si è visto, dichiarato senza esitazione la propria poca stima per il poeta ferrarese.

Per la nuova generazione di letterati filoclassicisti, Trissino era stato, secondo la testimonianza di Benedetto Varchi, un caposcuola. Varchi ricorda che quando era ancora "fanciullo", Machiavelli e altri "all'orto de' Rucellai ... udivano il Trissino e l'osservavano più tosto come maestro o superiore che come compagno"23. E Trissino non solo si era imbarcato nella stesura di un poema che si voleva aristotelicamente in antitesi allo stile dei popolari Romanzi, ma aveva nella sua Lettera di Dedica a Carlo $V$ affermato i principi estetici a cui il nuovo poema epico doveva attenersi, e cioè l'osservanza dei criteri estetici della Poetica aristotelica e l'imitazione del modello omerico 24 .

In effetti Trissino nella Lettera di Dedica a Carlo $V$ non si è pronuncia-

\footnotetext{
${ }^{22}$ Maggi, In eundem librum propriae Annotationes, 67. Sono i commenti del solo Maggi (rappresentano le riflessioni più recenti di Maggi dopo la sua venuta nell'ateneo ferrarese), incorporati, ma distinti dai precedenti, sotto ogni particella della Poetica aristotelica.

${ }^{23}$ Varchi, Lezione terza della poesia in Varchi, Opere, 2:718. Questo era nel 1513 (per la cronologia si veda Morsolin, Giangiorgio Trissino, 71 ss.).

${ }^{24}$ Musacchio. "Il poema epico ad una svolta: Trissino tra modello omerico e modello virgiliano".
} 
to affatto sul problema del narratore. Il fatto è sorprendente perché questo criterio avrebbe fornito il destro ad una critica di quella che era una pratica corrente nei Romanzi e ampiamente usata nel Furioso, e cioè il ruolo larghissimo assegnato alla voce del narratore, a cui si oppone il giudizio di Aristotele che, con la sua lode di Omero, invita al contrario a limitarlo quanto più possibile. E invece nella Lettera di Dedica a Carlo $V$ di questo aspetto non si parla affatto. Nel seguito, nelle Divisioni 5 e 6 della sua Poetica, in cui fornisce una parafrasi della Poetica aristotelica, riprodurrà semplicemente il passo del capitolo III ("il modo col quale devemo ... imitare ... è di tre maniere: l'una, che 'l poeta parla sempre in sua persona e non induce mai altre persone che parlino, come sono quasi tutte le elegie, le ode, le canzoni, e le ballate, e li sonetti e simili; l'altra è che 'l poeta mai non parla in sua persona, ma solamente induce persone che parlano, come sono comedie, tragedie, egloghe e simili; la terza è che "l poeta parte parla et enunzia e parte introduce persone che parlano, come sono gli eroici di Omero e di Vergilio e le cantiche di Dante et i Triomfi del Petrarca, e la nostra Italia liberata da' Gotti, e simili'); e quello del capitolo XXIII ("Omero poi in molte altre cose è degno di essere laudato, ma specialmente in questo che solo di tutti e' poeti conobbe quello che si dovea fare, cioè che 'I poeta dee dire poche parole da sé. Perciò che in quelle non è imitatore, e però egli poi che ha detto alcuni pochi versi subito induce o uomo o donna o altro che parli e dimostri il costume ...")25.

Sebbene Trissino riporti accuratamente i due passi aristotelici, egli non vi insiste sopra, e dà una larga scelta di esempi del modo misto che include con Omero anche Virgilio e menziona fra le opere contemporanee solamente il proprio poema epico. Il secondo passo, quello in cui Aristotele consiglia al narratore di mettersi nell'ombra, e che sarebbe potuto servire a denunziare la pratica dei volgari 'romanzi', non viene commentato e non è esemplificato in alcun modo ${ }^{26}$.

Nonostante il silenzio sorprendente di Trissino al riguardo, quello che si pensava a Firenze a proposito dei giudizi di Aristotele sulla opportunità di limitare il ruolo del narratore, e quello che di conseguenza si doveva pensare e giudicare del ruolo tradizionalmente assegnato al narratore nei Romanzi, e in particolar modo dell'uso che ne aveva fatto Ariosto nel

${ }^{25}$ Trissino. La Poetica (La Quinta Divisione e La Sesta Divisione) in Trattati di Poetica e Retorica del Cinquecento, vol. 2, pp. 13 e 48.

${ }^{26}$ Oltretutto Trissino nel suo proprio poema non è affatto alieno dall'impiegare una voce narrativa molto estesa (e dunque in contrasto alla spiccata preferenza aristotelica). 
Furioso, risulta chiaro dalle parole di Varchi in una lezione pubblica, Della Poetica in generale, datata Ottobre 155327.

Varchi non fa mistero della sua dipendenza da quello che "insegna nel principio della sua divina Poetica divinamente Aristotile”. Dopo aver accennato alla tripartizione dei modi della narrazione ("i poeti ... alcuna volta favellano essi sempre...come Virgilio nella Georgica....alcuna volta fanno che sempre favelli altri ... come fa quasi sempre il Petrarca. Alcuna volta parte favellano essi e parte introducono altri a favellare come fece Omero e Virgilio nell'Eneide e il Petrarca ne' Trionfi e Dante nella Commedia"), egli conclude: "Nella qual cosa dovemo sapere che quanto meno favella il poeta, tanto è l'imitazione maggiore, e conseguentemente più lodevole il poema"28. Ariosto non è menzionato specificamente in questo contesto, ma Varchi non tralascia una sola occasione di manifestare la sua poca stima per l'autore del Furioso ${ }^{29}$.Varchi è cosciente del fatto che la sua ostilità all'opera di Ariosto è talmente sistematica da apparire come una vera e propria campagna denigratoria. E, reagendo contro coloro che potrebbero criticare la sua insistenza ad abbassare il poeta ferrarese, se ne difende affermando che è mosso a "dire pubblicamente quello che" ha "privatamente detto altrove" spinto soltanto dal desiderio di "dire il vero". La critica principale sollevata da Varchi è naturalmente il mancato rispetto alla unità d'azione. Con Varchi, il quale univa ad una vasta cultura un mediocre acume critico, vediamo affermarsi in modo radicale il binomio di opinioni critiche assunto da quasi tutti i letterati del suo stampo, la cieca obbedienza alla lettera di Aristotele e il disprezzo per i risultati artistici del poeta ferrarese.

Più precisamente (e ancora prima delle critiche pubblicamente ostentate da Varchi in anni ancora più vicini al viaggio di Pigna a Firenze) l'esistenza di una serie precisa di critiche puntuali all'arte ariostesca veniva rivelata in uno scritto di Simon Fornari in difesa dell'Ariosto, la Apologia, pubblicata a Firenze nel 1549 e dedicata al duca Cosimo de Medici. Queste critiche tendono a sottolineare il clima antiariostesco di Firenze, probabilmente, ma non necessariamente, legato alle proposte antivirgiliane di

27Varchi si considerava vagamente discepolo di Maggi. Si veda il documentatissimo articolo di Pirotti, "Benedetto Varchi e l'aristotelismo del Rinascimento".

${ }^{28}$ Varchi, Della Poetica in generale in Varchi, Opere, 2:688. Sui giudizi fluttuanti di Varchi che nel 1539 aveva dichiarato al contrario "Virgilio ... e Omero seppero e insegnarono ogni cosa forse meglio d'Aristotile", si veda l'analisi di Pirotti (294 ss.).

29 Per Varchi il Giron Cortese di Alamanni è "molte volte superiore" al Furioso (Della Poetica in generale in Varchi, Opere, 2:691). 
Trissino: "Sono costretto ... di diffendere (Ariosto) ... d'alcune riprensioni et per dir più veramente false calunnie". Fornari rivelava la presenza nell'opinione critica dello stretto accoppiamento delle critiche antiariostesche con l'accettazione della poetica aristotelica: "Ne sarebbe a noi questa impresa di poca fatica, quando sì fatti riprensori prender si veggano per lor fautore Aristotele" 30 .

Anche Fornari naturalmente difende Ariosto dall'accusa principale rivolta all' Orlando Furioso, la mancanza di unità d'azione (Fornari afferma che in realtà c'era un'azione principale: "l'impresa d'Agramante contra Carlo Magno") 31 . Ma Fornari inoltre menziona (sempre fra le critiche indirizzate all'Ariosto negli ambienti classicheggianti già nel 1549) quella che ci interessa da vicino; "Accusano etiandio il nostro poeta come colui c' habbia fatto il contrario di quello che tanto loda in Homero Aristotile, ciò è che egli pochissime cose racconta come da sé, ma fa che altri le dica o huomo o donna o Dea che migliore gli viene". E controbatte: "Si risponde che nell'Ariosto se non ben così spesso, pur si vede che egli induce delle persone non solamente che tra se favellino e tentionino, ma che raccontino novelle intere" (38). Indicando inoltre quali siano le differenze tra il genere drammatico e il racconto epico, Fornari faceva notare che in quest'ultimo "largo campo deve esser concesso alla persona del poeta di favellare" (38). Ma Fornari, oltre a cercare scusanti per il poema ariostesco, si permetteva addirittura di attaccare il punto di vista di Aristotele e la sua preferenza per lo stile omerico, che invece viene giudicato da Fornari troppo 'basso' per il genere epico. Il genere epico (così come esemplificato da Virgilio, il suo massimo esponente) deve mantenersi nello stile alto, mentre con Omero l'epica rischia di scivolare nello stile comico: "e forse a Virgilio così dispiacque quel modo tenuto da Homero che tante e tante volte induce altrui che ragioni che par una commedia pure la sua". Per non scendere nel comico Virgilio 'drammatizza' meno e 'racconta' di più che Omero, e in effetti a parte il racconto fatto da Enea a Didone "tutto il restante egli è che 'I racconta e non altri” (38). Fornari insomma non solo difende Ariosto dalle accuse dei seguaci della poetica aristotelica, ma addirittura critica il giudizio di Aristotele riguardo alle ragioni dell'eccellenza di Omero: un'osservazione che, nel suo clima contemporaneo, non mancava di audacia.

A proposito del "campo concesso alla persona del poeta di favellare", Fornari acutamente distingue due aspetti del 'parlare da sé': "Se si riguarda poi a ragionamenti il che par che più tosto accenna Aristotile che 'I tenore

${ }^{30}$ Fornari, Apologia brieve sopra tutto l'Orlando Furioso, 31.

${ }^{31}$ Fornari, Apologia brieve sopra tutto l'Orlando Furioso, 34. 
delle lunghe narrationi (onde dice che per ciò vuole che "l poeta poche cose dica dalla persona sua imperoché in questo non è imitatore), io rispondo che in sì fatti parlari havuti da persone diverse, il poeta nostro non è men copioso che Virgilio, quantunque in ciò non così assiduo come Homero esser si vegga" (38-39). Due sono i tipi del 'parlare da sé', e non uno solo: i "ragionamenti" e i "parlari havuti da persone diverse". Una distinzione dunque tra la 'drammatizzazione' a cui si riferiva il giudizio di Aristotele (che seguiva l'analisi fattane da Platone), e la tradizione nei Romanzi di introdurre un narratore sovente garrulo e sentenzioso; una distinzione che raramente i critici dell'Ariosto avranno presente. Per quanto riguarda i "parlari", Fornari, come si è visto, ritiene che Ariosto sia "non men copioso che Virgilio" e fortunatamente "non così assiduo" come Omero. Quanto ai "ragionamenti", Fornari li giudica positivamente perché offrono il destro al poeta di introdurre insegnamenti morali. Non solo infatti "suole l'Ariosto ... in molti lochi favoleggiando occultare qualche morale" (108), ma precisamente nei discorsi del narratore, secondo Fornari, Ariosto adempie alla funzione assegnata al poeta da Orazio di unire l'utile al dilettevole: "Poche sono gli principii de canti nella presente opera ne quali il poeta non si spatii dolcemente per alcun loco morale secondo che la suggetta materia richieda" (181).

Le risposte di Fornari alle critiche mosse al Furioso rivelano dunque la forza dei sentimenti negativi nutriti in Toscana a proposito di un genere letterario che, sviluppatosi in un'altra area geografica e culturale, non era riuscito ad ottenere la sua legittimazione negli ambienti letterari fiorentini. È dunque verosimile che Pigna avesse udito dalla bocca di questi ultimi anche l'ulteriore accusa ad Ariosto di aver fatto troppo larga parte alle parole del narratore, e questo in occasione di quel suo viaggio in Toscana che è all'origine della lettera indirizzata a Giraldi circa "i morditori" dell'Ariosto.

Ma non solo in Toscana il tono della critica era generalmente ostile all'autore del Furioso, anche a parte la questione della lingua ${ }^{32}$.

${ }^{32}$ Weiberg osserva che "The existence of the apologia (lo scritto di Fornari) presupposes a body of negative opinion, of attacks ... a body of contemporary objections to Ariosto which we cannot now consult directly", A History of Literary Criticism in the Italian Renaissance, I, 954. Anche in Toscana, naturalmente, non mancavano difensori dell'Ariosto, fra i quali, e in prima linea, troviamo Galileo, ammiratore incondizionato dell' Orlando Furioso (si vedano le sue Considerazioni al Tasso). Non vi è dubbio tuttavia che i 'professionisti' della letteratura (di contro ai semplici 'fruitori', cioè il "volgo" secondo l'espressione trissiniana) condannassero le pratiche dei Romanzi tanto meglio esemplificate dal Furioso in quanto esso era cosi popolare. Se si tiene conto dunque delle autorevoli voci 
Padova era un altro vigoroso centro di aristotelismo antiariostesco. Ne fa fede una testimonianza del diciannovenne Torquato Tasso, studente in quell'ateneo, il quale indirizzandosi nel 1562 ai lettori del suo Rinaldo, indica succintamente le direttive estetiche che aveva inteso seguire nella scrittura del suo poema giovanile. La sua argomentazione è un curioso esercizio di acrobazia perché l'autore del Rinaldo tenta di non alienarsi la simpatia degli ammiratori di Ariosto, ma al tempo stesso neanche quella dei classicisti denigratori di quello. Circa il problema sollevato da Aristotele a

critiche emananti dai centri ufficiali della cultura non si può non rimanere perplessi davanti alla proclamata tesi centrale del volume di Javitch (Proclaiming a Classic), secondo cui il poema di Ariosto avrebbe subito un non ben definito processo di "canonizzazione". La stranezza di questo titolo e della tesi martellata in particolare nella Introduzione riflette le preoccupazioni della critica americana contemporanea su un presunto 'Canone', ed è perfettamente incongrua quando venga inserita nell'analisi della guerra dei 'gusti' e degli 'stili' del '500 letterario italiano. Se si prescinde dall'assurda tesi, l'attento e pregevole studio di Javitch mostra come il successo popolare e editoriale del Furioso abbia saputo validamente contrastare il lavoro di demolizione menato dai classicisti. Ma indirettamente persino il tono difensivo assunto dai difensori di Ariosto dimostra che di tutt'altro si tratta che di un processo di canonizzazione. Il parere ufficiale ("gli huomini dotti et eruditi", secondo l'espressione usata da Malatesta in Della Nuova Poesia) condannava il Furioso, ma a questa condanna il piacere dei suoi lettori non poteva rassegnarsi, per cui in vari modi si procedeva a trovare scuse per le pecche stilistiche dell'opera. Javitch stesso, lungi dal nascondere questo dato di fatto, lo mostra anzi con un accurato studio delle reazioni critiche al Furioso. Non si capisce pertanto perché questo processo di sconfessione del romanzo di Ariosto da parte della critica imperante possa venir trattato come una "canonizzazione". E sempre a proposito di questa fantomatica canonizzazione, non bisogna dimenticare il frazionamente letterario dell'Italia: il fenomeno della controversia sul poema dell'Ariosto era limitato al settentrione con epicentro a Ferrara, non interessava larga parte del mondo letterario italiano. La questione della lingua era naturalmente in primo piano. A Firenze Fornari aveva nel 1549 difeso Ariosto per il fatto di prendere a prestito da "altre lingue" (Simon Fornari, Apologia brieve sopra tutto l'Orlando Furioso, 43-44). A Venezia, già nel 1544, Ludovico Dolce in una lettera indirizzata all'editore Gabriele Giolito, premessa alla sua edizione dell' Orlando Furioso e datata 1 marzo 1544, lo ringraziava per aver voluto pubblicare un'edizione corretta del capolavoro ariostesco e si scagliava contro "quelli che da propria temerità mossi ardiscono di emendare". I temerari avevano, in questo caso, accusato Ariosto per l'impiego di espressioni antiquate (Ludovico Dolce, Lettera a Gabriele Giolito). E Dolce continuava affermando che quei temerari "avrebbero dovuto esser trattati come peggio di criminali". Tra le parole che avevano voluto emendare, Dolce menzionava "unquanco", "guari", "huopo", "altresi”. 
proposito di quanto il narratore debba scrivere 'da se', Tasso, nel corso di un pur brevissimo scritto, vi fa riferimento ben tre volte. Nella sua prefazione al Rinaldo, Tasso accenna alle "severe leggi d'Aristotele" affermando di averne seguito il precetto che prescrive di "introducendo a parlar altri, spogliarsi de la persona di poeta". Vuole tuttavia riuscire a ingraziarsi sia $\mathrm{i}$ "severi filosofi seguaci d'Aristotile, i quali hanno innanzi gli occhi il perfetto esempio di Virgilio e d'Omero", sia "i troppo affezionati dell'Ariosto" (5). Tasso afferma dunque, in un contorcimento straordinario, di non aver usato "ne' principi de' canti quelle moralità, e que' proemi ch'usa sempre l'Ariosto" (5), conscio di quello che "Aristotile chiaramente dica ne la sua Poetica ... che tanto il poeta è migliore, quanto imita più, e tanto imita più quanto men egli come poeta parla e più introduce altri a parlare"; ma invece di vantarsi di questa sua adeguazione all'invito di Aristotele, se ne scusa come di una colpa. E la sua giustificazione per aver seguito il precetto di Aristotele, che pure altrove dichiara dover essere seguito, consiste nell'appoggiarsi ad un giudizio di Pigna, proprio quell'affermazione con la quale Pigna aveva voluto discolpare Ariosto dall'accusa di aver peccato contro il precetto di Aristotele: "io credo che vero sia , ciò che il dottissimo signor Pigna dice in questa materia, che l'Ariosto tai proemi non avrebbe fatto, se non avesse stimato che, trattando di vari cavalieri e di varie azioni ... gli era necessario render talvolta docili gli auditori ... congiungendo le cose che s'hanno a dire con quelle che gia dette si sono". Ora, proseguiva Tasso, il suo Rinaldo aveva una sola azione e pertanto non necessitava di quell'infrazione al codice aristotelico che invece era giustificabile in Ariosto $^{33}$.

Non commetteva in effetti l'infrazione nel suo Rinaldo, che appunto non include nella sua narrativa alcun commento del poeta, e dunque si conforma perfettamente al criterio aristotelico. A giustificazione di quelli che invece avevano peccato contro quel criterio, il giovane Torquato faceva notare che non solo Ariosto ma anche suo padre Bernardo Tasso aveva così composto il suo Amadigi.

Il problema così vivo nella prefazione al Rinaldo perderà in seguito importanza agli occhi di Tasso. Nei Discorsi dell'Arte Poetica pubblicati nel 1587 ma scritti probabilmente negli anni '60, i due modi del racconto sono chiaramente delineati: "Il modo è il narrare e il rappresentare: narrare è ove appar la persona del poeta, rappresentare ove occulta è quella del poeta e appare quella de gli istrioni", "Imita il romanzo e l'epopeia con l'istessa maniera: nell'uno e nell'altro poema vi appare la persona del poeta;

${ }^{33}$ Tasso. Rinaldo, 4-6. 
vi si narrano le cose, non si rappresentano" 34 . Ma la questione cruciale dell'estraneità della voce del Narratore al processo mimetico non è in alcun modo menzionata. E ugualmente slegate da un giudizio sulla qualità mimetica e pertanto artistica dei due modi saranno le menzioni nei più tardivi Discorsi del Poema Eroico, del 1594, "la poesia in duo modi cerca di mostrarla (la bellezza) e di porcela davanti a gli occhi: l'uno è la narrazione, l'altro la rappresentazione; e l'uno e l'altro è contenuto sotto la imitazione" (71), distinzione a cui fa di nuovo riferimento ${ }^{35}$. E anche nel nutrito epistolario con Scipione Gonzaga e e con Luca Scalabrino degli anni 1575-76, in cui Tasso dichiara le sue preoccupazioni teoriche, e il nome di Aristotele e della sua Poetica sono tutt'altro che infrequenti, lo specifico problema dell'importanza del 'drammatico' sul 'narrativo' non viene menzionato, neppure quando nella lettera a Gonzaga del 4 ottobre 1575, Tasso afferma che Virgilio rispetto a Omero "si ristringe alla narrazione, lasciando l'imitazione" 36 . Nel corso del lavoro di creazione sui suoi poemi, Tasso si sarà forse reso conto che il problema della voce del Narratore era più complesso di quanto i seguaci di stretta osservanza della Poetica aristotelica sembrassero credere.

Al tempo del Rinaldo tuttavia il giovane Torquato intendeva seguire strettamente i precetti aristotelici. E faceva inoltre i nomi degli studiosi che a Padova avevano insegnato appunto quei precetti: Sigonio e Sperone Speroni.

Per quanto riguarda Carlo Sigonio, Torquato Tasso accenna al suo insegnamento della Poetica di Aristotele nell'ateneo padovano ("Poetica, la qual ora con gloria con gloria di sé e stupore e invidia altrui, espone in Padoa l'eloquentissimo Sigonio") ${ }^{37}$. Di quale tipo di insegnamento si trattasse, non è facile rendersi conto a partire dai suoi scritti, perché Sigonio appare nei suoi commenti sul trattato aristotelico interessato unicamente da questioni filologiche nella prosecuzione della sua violenta polemica con Robortello. Sigonio si difende enfaticamente dagli attacchi di Robortello ("Robortelle ... contumeliis me continuo tuis onerabis ?"). A proposito poi del passo nel capitolo III della Poetica di Aristotele che introduce la distinzione tra drammatizzazione e racconto puro e semplice, Sigonio critica l'interpretazione che Robortello ha dato della espressione EN TOIS

${ }^{34}$ Tasso, Discorsi dell'arte poetica e del poema eroico, 11 e 27.

${ }^{35}$ Tasso, Discorsi dell'arte poetica e del poema eroico, 101 e 130.

${ }^{36}$ Tasso, Lettere poetiche, Lettera XXVIII, 249-250.

${ }^{37}$ Tasso, Rinaldo, 4-6. 
AUTOIS (1448a20). Per Robortello Aristotele vuol dire "con gli stessi uomini", per Sigonio (indubbiamente a ragione se si guarda al contesto; si vedano a proposito i commentatori moderni) vuol dire "con gli stessi strumenti"38.

Tenendo conto anche di quello che risulta dalla direzione delle ricerche, essenzialmente di natura storica, a cui in seguito si dedicherà Sigonio, sembrerebbe probabile che anche il suo insegnamento sulla Poetica abbia essenzialmente insistito sui problemi della retta interpretazione dell'antico testo piuttostoché, come invece nel caso di Trissino, su una interpretazione volta a segnalare e incoraggiare un cambiamento nel gusto letterario della propria epoca.

Diverso è invece il caso di Sperone Speroni. Il sospettosissimo autore della Canace si piccava di essere un gran conoscitore di questioni teoriche. Anzi in una lettera del 1581 accusava Torquato di avergli rubato le idee e di averle rese pubbliche sotto il proprio nome in uno scritto : "interrogato molte fiate dal Tasso e rispondendoli io liberamente ... egli n'ha fatto un volume e mandato al Sig. Scipione Gonzaga per cosa sua e non mia" ${ }^{39}$.

Per buon senso (o forse semplicemente per mancanza di interesse) Speroni si dimostra un aristotelico non fanatico: "Se Aristotile veniva al mondo dopo Virgilio, egli formava la sua poetica non dalla Iliade, come egli fece, di quello Omero, ma dalla Eneida solamente, benché latina la ritrovasse". Apparteneva peraltro all'esiguo plotone di quelli che ammiravano Omero più che Virgilio: "In summa, senza Omero era niente Virgilio, quanto alla disposizione e favola, ma le orazioni son ben sue, ed in questo fu brevissimo e sentenzioso, avendo in ciò forse più dell'istorico che del poeta ... Virgilio narra bene ma non bene imita né le guerre né le fortune"40; "dalla dilettazion di Omero nasce allegria ed ilarità, da quella di

38 Sigonio, Emendationum, Liber II, cap. XXXIII, 102 e 211 . Sulla controversia di Sigonio con Robortello si veda Tiraboschi, Storia della letteratura italiana, vol. 7 , pt 3, 212 ss.

${ }^{39}$ Speroni, Epistolario, 272. Si tratta evidentemente dei Discorsi dell'arte poetica, che Speroni doveva conoscere nella versione manoscritta dato che verranno pubblicati solo nel 1587. Forse a questa (o a simile accusa) Tasso si riferiva nei successivi Discorsi del poema eroico, pubblicati nel 1594, quando dichiarava:"ho dubitato che altri non potesse credere ch'io volessi attribuirmi l'opinione d'alcuni" (Tasso, Discorsi dell'arte poetica e del poema eroico, 62).

${ }^{40}$ Speroni, Dialogo primo sopra Virgilio, 127. Ma ci si puo chiedere quanto chiare fossero le sue idee a proposito dei concetti aristotelici in materia di poetica. Muovendosi nella direzione della concezione platonica finisce per osservare "la poetica non è proprio arte ma furore”, 576). 
Virgilio nasce stupore e melanconia, il che non è proprio del poeta ma anzi dell'istorico" 41 ; "conobbe dunque Virgilio dopo fatta la Eneide le sue imperfezioni comparandola a Omero ... Vistosi adunque nelle medesime cose trattate da lui e da Omero, ad Omero non esser aggiunto imitandolo, ma esserli secondo..."42. In numerosi scritti su Virgilio (due Dialoghi sopra Virgilio, di cui il secondo non è finito, e otto Discorsi sopra Virgilio) aveva spulciato spietatamente tutte le pecche che aveva creduto trovare nell'autore dell' Eneide, linguistiche, storiche e anche vagamente riferentesi ai precetti aristotelici: "le orazioni e narrazioni di Virgilio e descrizioni poetice son nobilissime, ma non imita come dovrebbe, ne è florido e ornato come dovrebbe ed ha piuttosto dell'istorico che del poeta" 43 .

Non meno severo era al riguardo di Ariosto. In una lettera a Bernardo Tasso, nel 1559 Speroni dichiara: "Ariosto il cui poema si può agguagliare a una donna che ha poche parti che belle siano, solamente ha un non so che onde ella piaccia alla gente; e forse in lui quel che non so che, si sa e sente che sia e non è suo ma d'altrui, cioè a dire che la invenzione e la disposizione di quella opera con i nomi de' cavalieri fur di colui cui egli sdegna di nominare o per dir meglio non osa". Ariosto insomma, secondo Speroni, avrebbe rubato a Boiardo tutte "le poche parti che belle siano" 44 Nel suo dialogo, Della Nuova Poesia, Malatesta introdurra come partecipante uno Speroni appassionato difensore dell'Ariosto, e ben diverso dallo Speroni rivelato in queste sue sprezzanti parole. Nel dialogo di Malatesta altri partecipanti alla discussione sostengono invece che la popolarità è indice di eccellenza. E alla fine i partecipanti discuteranno se Platone avrebbe accettato Ariosto nella sua Repubblica.

Sebbene sia convinto di aver insegnato tutto a Tasso sul poema eroico, in realtà Speroni non sembra aver avuto interessi o capacità di ordine critico per quel che riguardava il genere epico; e in particolare sul problema del ruolo del narratore non riesce a precisare le sue idee, anche se a questo sembra si riferisca quando parla del carattere non 'imitativo' dello stile virgiliano.

Gli interessi di Pietro Vettori erano dello stesso ordine di quelli di Sigonio. Nei suoi commenti alla Poetica aristotelica del 1560, Vettori si

${ }^{41}$ Speroni, Discorso primo sopra Virgilio, 435 e 439.

${ }^{4}$ Speroni, Discorso quinto sopra Virgilio, 532.

${ }^{43}$ Speroni, Discorso primo sopra Virgilio, 438.

${ }^{44}$ Speroni, Epistolario, 68-69. 
sforza di trovare il senso esatto delle parole dell'autore, con acume, intelligenza e una vasta cultura, ma senza che vi interferiscano le preoccupazioni contemporanee per il cammino futuro della scrittura epica. I due modi del racconto sono chiaramente identificati: "commemorantem, vel aliud quippiam sive personam sive rem factum" e "eundem manentem et non se immutantem"; ne nasce il modo misto (esemplificato da Omero e Virgilio) "poeta enim illic quaedam sua ex persona exponit: alia autem narrat alius factus". È inoltre chiaramente identificato il processo narrativo in Omero:" poeta enim illic quaedam sua ex persona exponit, alia autem narrat alius factus: alius enim fit poeta cum induit personam alicuius, et quaecunque ipse loquitur ex ore illius prodire fingit". E Vettori nota che Aristotele giudica ad un certo punto il modo narrativo non imitativo: "Nam in epico carmine poeta cum exponit sua ex persona aliquid, proprie neminem imitatur" 45 . Ma questo sorprendente scarto dello Stagirita dalla sua generale concezione sulla natura imitativa della poesia non è sottolineato e non viene commentato da Vettori, il quale anzi non sembra neppure averne colta la contraddizione (così come gli altri grandi commentatori prima di lui).

Di ben altra tempra critica, e risolutamente antiariostesco, è il modenese Castelvetro. Nella sua Poetica d'Aristotele vulgarizzata et sposta, del 1570, Castelvetro è convinto assertore della poetica aristotelica (anzi non si potrebbe trovare un critico che meglio corrisponda alla definizione tassesca di "severo filosofo seguace d'Aristotile"). Infatti, mentre l'autore del Rinaldo si atteneva al precetto di limitare al massimo gli interventi del "poeta" ed eliminare i "proemi" e al tempo stesso li scusava in Ariosto accettando le ragioni per la loro presenza nell' Orlando Furioso avanzate da Pigna in I Romanzi, Castelvetro più conseguentemente condanna invece senza appello Ariosto il quale era caduto nell'errore del poeta che "posposto l'ufficio di narratore che era suo proprio, imprenda l'ufficio di predicatore et di correggitore de' costumi fuori di tempo". In questo errore, aggiunge Castelvetro, "non cade mai Homero, ma sì Virgilio alcuna volta ... et più spesso di lui vi cagiono quelli poeti che sono meno buoni di lui ... et più spesso di tutti Ludovico Ariosto nel suo Orlando Furioso". Che poi si tratti di un errore, e anzi "che non è errore da stimare poco", Castelvetro lo stabilisce chiaramente sulla base delle affermazioni di Aristotele: "pare che si possa conchiudere che Homero, il quale introduce spesso le persone a favellare e attribuisce loro le favelle lunghe et usa la materia dell'attione particolareggiata, sia rassomigliatore et per con-

${ }^{45}$ Vettori, Commentarii in primum librum Aristotelis de arte poetarum, 25 e 26. 
seguente poeta, et che Virgilio introducendo poche volte persone a favellare et assegnando loro favella brieve più assai che non fa Homero et usando materia d'attione universaleggiata, sia non rassomigliatore et per conseguente non poeta. Se il poeta in quella parte dell'epopea nella quale narra solamente et racconta l'attione et non introduce persona a favellare non è rassomigliatore secondo Aristotele et per conseguente non è poeta, che diremo noi del poeta in quella parte dell'epopea nella quale egli né narra attione né introduce persona a favellare, ma giudica le cose narrate o riprendendole o lodandole o tirandole ad utilità commune et ad insegnamenti civili et del ben vivere ? Certo non altro se non che egli non è rassomigliatore né per cagione del modo non introducendo persona niuna a favellare, io dico persona niuna di quelle che intervengono nella favola, né per cagione della materia della favola, essendo questa materia seperata dalla favola et nascente dall'affettiona del poeta verso l'attione. Hora se egli non è rassomigliatore né per cagione del modo né per cagione della materia, seguita che anchora non sia in questa parte poeta" 46 .

Pur senza menzionare esplicitamente Ariosto come uno dei contravventori del giudizio aristotelico riguardo agli interventi degli autori, Alessandro Piccolomini, nelle sue Annotazioni, del 1575, si allinea sulle posizioni di Castelvetro. Piccolomini condanna gli autori epici che intervengono con i loro giudizi sullo svolgimento della storia. Spiegando il passo dal XXIV capitolo della Poetica, Piccolomini afferma che la condanna aristotelica non riguarda "ogni parlare" del poeta "ma solamente quando lo fa in persona propria spogliato nondimeno della persona del poeta". E, riassumendo, distingue in Aristotele tre modi narrativi: "l'un è quando egli si veste della persona di chiunque sia et imitando con le parole le parole di quella ... Un altro modo è quando egli con le parole imitando non le parole ma le cose over $\mathrm{i}$ fatti in propria persona come poeta narra li detti fatti ... Un altro finalmente modo sarà poi quando il poeta spogliandosi l'habito di poeta non come narratore, ma come giudicatore e stimatore delle cose narrate e come (insomma) interessato parla ... non come imitatore ma come giudicatore, il giuditio et il concetto suo interponendo" 47 .

Mentre Castelvetro esamina la distinzione fra "narrazione semplice" e "narrazione mimetica" nella prospettiva del diverso stile impresso in un'opera letteraria dal suo autore, altri studiosi platonizzanti o aristotelizzanti, negli ultimi decenni del '500, pur analizzando attentamente la stessa distinzione, si mostrano insensibili a questa dimensione della questione.

${ }^{46}$ Castelvetro, Poetica d'Aristotele vulgarizzata et sposta, 301b-302a

47 Piccolomini. Annotationi, 385. 
Segni, nelle Lezioni intorno alla Poesia del 1573, riprende i due modi della narrazione proposti da Platone e ribaditi da Aristotele, ma obietta che, mentre Platone chiarisce cosa sia l'imitazione, Aristotele invece non lo spiega affatto. In ogni caso la questione dei due modi della narrazione è giudicata da Segni in una prospettiva decisamente platonica: "la imitazione poetica ... è parlare e dire qualunque cosa sotto l'altrui persona" ma "non è cosa sì bella né onorevole uscir del proprio abito e vestirsi l'altrui" e "la maestà del poeta ... si restrigne et è ... ridotta a parlare in persona d'altri e far il mimo". Come è possibile dunque salvare il valore dell'arte se la poesia è imitazione? Una parziale soluzione è trovata chiarendo i modi differenti in cui l'imitazione viene fatta e gli oggetti differenti di tale imitazione, anche se Segni manterrà la sua riprovazione di matrice platonica per la "non molta dignità veramente de la poesia poiché l'essenza sua è tale che quando più ne partecipa et è poesia, tanto più perde di bontà e d'onore, e quanto più si discosta da lei e meno de la sua essenza ritiene, tanto più da' valentuomini è lodata e tenuta cara" 48 . Il problema che Segni si pone e crede di aver risolto è comunque d'ordine filosofico, e non riguarda la discussione che ha violentemente diviso critici e poeti della precedente generazione a proposito del modo corretto di comporre un poema eroico.

Parzialmente d'accordo con le conclusioni di Castelvetro si dichiara Patrizi, nonostante il fatto che, in quanto nemico dichiarato di Aristotele e convinto platonista, si trovava sull'opposto versante ideologico. Patrizi dedica un intero capitolo della Deca Disputata del 1586 alla nostra questione, citando in intero il passo dalla Repubblica di Platone e i passi dalla Poetica aristotelica. $\mathrm{Ma}$, annegate come sono nella sua naturale verbosità ${ }^{49}$, non è facile comprendere quali siano le sue posizioni, negative in questo caso non solo nei riguardi di Aristotele ("de' modi dell'imitare né a compiuto né vero fu da Aristotile trattato") ${ }^{50} \mathrm{ma}$, eccezionalmente, anche di Platone. Ancor meno chiara la posizione di Francesco Caburacci, il quale nel suo Breve Discorso in difesa dell'Orlando Furioso, del 1580, menziona l'obiezione fatta ad Ariosto dai fedeli della Poetica aristotelica a proposito del ruolo del narratore "che gli s'apparteneva di parlar poco in persona propria", ma in effetti senza che la critica venga rintuzzata ${ }^{51}$.

\footnotetext{
${ }^{48}$ Segni. Lezioni intorno alla poesia, 21-22 e 52-53.

${ }^{49}$ Tiraboschi lo definiva "intollerante di freno" (Tiraboschi, Storia della letteratura italiana, vol. 7 , pt. 3, 308).

50 Patrizi da Cherso, Della poetica, 195.

${ }^{51}$ Caburacci da Imola, Trattato dove si dimostra il vero et novo modo di fare le imprese, 75 .
} 
E anche Mazzoni, un pensatore invece fortemente influenzato da Aristotele, sembra incapace di cogliere quanto la distinzione sia importante per l'indirizzo compositivo di un'opera. Mazzoni, nella Introduzione alla Difesa della Commedia di Dante, del 1587, si sforza di provare che i due grandi pensatori dell'antichità, Platone e Aristotele, sono in pieno accordo sul fatto che il racconto, tanto nella forma drammatizzata che in quella semplicemente narrata ("rappresentando le persone stesse che dicono" o "raccontando solamente le cose in persona del poeta" o "parte raccontando e parte introducendo altri a dire") è sempre e solo una imitazione ${ }^{52}$.

Verso la fine del secolo l'esistenza di una dicotomia rispetto al modo in cui il racconto può essere svolto è ormai un insegnamento universalmente assorbito, ma la forza che questa distinzione esercitava nella determinazione di due stili, e dunque di due gusti letterari opposti, sembra essersi perduta.

I termini impiegati variano da critico a critico, ma è chiaro che il dibattito rinascimentale sui due modi narrativi portati alla luce da Platone e da Aristotele ha perfettamente colto a segno l'essenza della distinzione, quella distinzione sulla quale, a distanza di secoli, la moderna narratologia ha posto largamente l'accento contrapponendo il 'narrare' al 'mostrare', la descrizione della scena nelle parole dell'autore e la diretta presentazione degli eventis3.

Tuttavia, se il problema dei due modi narrativi è stato perfettamente messo a fuoco dai letterati del XVI secolo, notevoli ambiguità sono riscontrabili nell'impiego che ne hanno fatto quando intendevano indicare il cammino che, secondo loro, il racconto epico avrebbe dovuto imboccare nella fase che si apriva dopo Ariosto. E mentre nella definizione delle due contrastanti maniere di narrare, le parole dei letterati rinascimentali sono perfettamente chiare, le varie dimensioni della questione del ruolo del narratore sono invece sovente confuse.

Ovviamente il problema così come Platone e Aristotele se lo erano posto, era più semplice. Consisteva nella pura e semplice identificazione di due tecniche del racconto a partire dall'opera del più illustre dei narratori greci, l'autore dell'Iliade. Omero, come Aristotele ribadiva, è un maestro dello stile mimetico.

Nello stile mimetico il narratore si sforza di scomparire; più è abile nel

\section{Mazzoni. Introduzione alla difesa della 'Commedia' di Dante, 32 ss.}

53"Telling and showing" sono i termini inglesi, ormai entrati nel vocabolario della critica per merito di Lubbock in The craft of fiction. Ma si vedano le penetranti analisi di Booth The Rhetoric of Fiction; e inoltre le osservazioni di Genette nei capitoli 4 e 5 del suo Discours du récit, 183-267. 
'mostrare' e meno appare come colui che racconta. E la tecnica più semplice per effettuare il racconto 'mimetico' in contrapposizione al racconto 'diegetico' consiste in un largo impiego del discorso diretto. Proprio questa caratteristica era stata messa in luce nell'esemplificazione fornita da Platone. Nella riscrittura del passo omerico il discorso diretto di Crise ("pregava tutti gli Achei ... : 'Atridi ... a voi diano gli dei"”) viene trasformato da Platone in un discorso indiretto ("pregò che a loro gli dei concedessero"). E anche Aristotele si era riferito al medesimo aspetto della tecnica mimetica quando lodava Omero che "subito introduce" un personaggio che si esprime con le proprie parole. La narrazione che predilige il 'mostrare' rispetto al 'narrare' fa parlare direttamente i vari personaggi invece di riferirne i discorsi mediatamente nelle parole del narratore ${ }^{54}$.

$\mathrm{Ma}$, a differenza di Omero che predilige il 'mostrare' e impiega ampiamente il discorso diretto, Virgilio (il grande modello epico per i letterati rinascimentali), pur impiegando sovente il discorso diretto, chiaramente è un autore che preferisce la tecnica del 'racconto puro e semplice', la tecnica 'diegetica'. E, naturalmente, se la tendenza del narratore che, come Omero, predilige la 'mimica' (e dunque 'mostra' e non 'narra') è quella di scomparire come tale, il narratore che preferisce la 'diegesi', e dunque usa il 'racconto puro e semplice', è sempre scopertamente presente nella sua narrazione. Infatti "se c'è un racconto, ci deve essere una persona che racconta, una voce che narra"55.

${ }^{54} \hat{\mathrm{E}}$ chiaro comunque che in realtà il narratore è inevitabilmente presente. Come dice bene Genette "La forme la plus 'mimétique' est évidemment celle que rejette Platon, où le narrateur feint de céder littéralement la parole à son personnage" (Discours du récit, 192): dove è da sottolineare la parola "finge". E pertanto, anche in un'opera eminentemente mimetica come l'Iliade, il narratore appare fin dai primissimi versi ("Cantami"), indirizzandosi direttamente all'uditorio ("Chi per primo ...?”). E anzi può inaspettatamente farsi avanti e, abbandonando la sua neutralità, prorompere in giudizi personali (per esempio con quel sorprendente "Stolto!" affibbiato ad Agamennone che, nell'inizio del Canto Il, ha ritenuto veritiero il sogno inviatogli da Zeus e crede scioccamente nella immediata caduta di Troia); o commentare cinicamente, al momento della morte di Àssilo, nel Canto VI, che sebbene costui avesse molti amici nessuno di loro si interpose a fare da scudo all'arma di Diomede. E, incidentalmente, questi esempi smentiscono l'affermazione di Castelvetro secondo cui Omero "non cade mai" nell'errore di permettere al narratore di dare giudizi o fare commenti (Castelvetro, Poetica d'Aristotele vulgarizzata et sposta, 302).

${ }^{55}$ La felice espressione è di Chatman: "Is the statement directly presented to the audience or is it mediated by someone-the someone we call the narrator? Direct presentation presumes a kind of overhearing by the audience. Mediated 
Ecco dunque perché, mentre Platone e Aristotele avevano un compito relativamente lineare nel delineare i due modi narrativi e nell'indicare la loro divergente inclinazione estetica, dato che esemplificavano a partire da un unico e monolitico modello, i critici rinascimentali si trovavano invece davanti ad un contesto più complesso. Le nozioni critiche che volevano adottare erano quelle di Aristotele, grande ammiratore di Omero, ma il modello poetico a cui si identificavano era essenzialmente Virgilio, e solo secondariamente e derivativamente Omero. E non solo Virgilio e Omero sono autori le cui tecniche narrative sono diversissime, ma l'opera virgiliana poco corrisponde ai criteri aristotelici.

Questa differenziazione nel ruolo assegnato al narratore (la voce del narratore onnipresente dell'Eneide, e quella più dissimulata dell'Iliade) i critici rinascimentali non potevano ovviamente non averla percepita. E tuttavia molti di loro hanno insistito a pretendere che Virgilio e Omero presentavano somiglianze fondamentali anche sotto questo aspetto; e, nella loro ossessione di ritrovare una classicità univoca, hanno sovente insistito ad arruolare Virgilio nella schiera degli scrittori che applicano il canone aristotelico della massima mimesi. Così Robortello ha estratto proprio da Virgilio gli esempi per lo stile 'mimetico', incapace di vedere la distanza che esiste tra le due estetiche o refrattario ad accettarla. E Varchi ugualmente associa Omero e Virgilio come poeti che allo stesso modo "introducono altri a favellare". Ora è ovvio che Aristotele non avrebbe sicuramente dichiarato di Virgilio che "dopo poche parole come di presentazione, subito introduce o un uomo o una donna (a parlare)". Ma, nel fervore della polemica pro e sopratutto contro la letteratura dei Romanzi, i letterati del Rinascimento non si sono preoccupati di identificare la specificità del narratore nei due differenti modi narrativi, mettendo insieme le varie forme di intervento, da quello dell'autore cosidetto 'implicito' a quello di un personaggio che si presenta come il Narratore.

I critici che insistevano a voler associare Virgilio a Omero sulla base della quantità di discorsi diretti nelle loro rispettive composizioni, riuscivano a trovare un numero adeguato di esempi nell' Eneide perché confondevano due tecniche diverse di scrittura. Da una parte la scelta della tecnica mimetica insiste a 'mostrare', ponendo le parole del discorso direttamente nella bocca stessa dei personaggi. D'altra parte la tecnica dello

narration, on the other hand, presumes a more or less express communication from narrator to audience. This is essentially Plato's distinction between mimesis and diegesis, in modern terms between showing and telling. Insofar as there is telling, there must be a teller, a narrating voice" (Story and Discourse, 146). 
'incastro narrativo' pone un racconto dentro l'altro, facendo di un personaggio il narratore di un suo racconto. A questa seconda tecnica (e non alla prima) appartiene il racconto della distruzione di Troia in bocca di Enea, in tutto il II Libro dell' Eneide ${ }^{56}$. Si tratta in questo caso di un racconto dentro un racconto, ma non riguarda la tecnica della narrazione mimetica. E dunque a torto viene enumerato fra gli esempi del modo mimetico, a cui in effetti non appartiene.

La convinzione dell'esistenza di un modello classico unitario rappresentato insieme dall' Iliade e dall' Eneide era fondata su una volontaria miopia. E di conseguenza, necessariamente ambigua risultava la proposta di un nuovo corso che il poema eroico post ariostesco avrebbe dovuto seguire conformandosi ai criteri di un fantomatico modello omerico-virgiliano. Era un equivoco che alcuni pochi degli stessi critici rinascimentali hanno chiaramente denunziato.

La cosa non era sfuggita a Trissino, critico acuto quanto mediocre poeta epico, il quale, pur senza porla in riferimento al problema del narratore, aveva colto la divergenza dello stile virgiliano da quello omerico, giudicando negativamente l'ambizione del primo ad una eccessiva "altezza" e lodando invece la preoccupazione per ottenere 'vividezza' nella narrazione (la ENARGEIA) del secondo ${ }^{57}$.

Anche Fornari, come si è visto, sottolinea con precisione quella differenza tra i due grandi modelli classici, ma opta poi per Virgilio perché Omero, con il suo stile 'mimico' "tante e tante volte induce altrui che ragioni che pare una commedia”.

Castelvetro, al contrario, sul medesimo divario sulle tecniche narrative nei due poemi, giudicava invece che Virgilio pecca contro le regole di Aristotele perché introduce "poche volte persone a favellare" e dunque non è "rassomigliatore e per conseguente poeta", condannando così lo stile virgiliano.

Senza entrare nel merito della questione della validità poetica dell'uno o dell'altro modo, in un trattato del 1588 , Breve discorso intorno alla narrazione poetica, Verdizzotti ha elaborato un sistema di quattro modi narra-

${ }^{56}$ Una tecnica, incidentalmente che Ariosto usa regolarmente: "una tecnica usata volentieri dall'Ariosto nel Furioso" (Segre, "Testo Letterario, interpretazione, storia: linee concettuali e categorie critiche", 34).

57È un'altra faccia, ovviamente, dello stile mimetico. Per la posizione critica di Trissino si vedano: Musacchio "Il poema epico ad una svolta: Trissino tra modello omerico e modello virgiliano" e "Il 'principio, mezzo e fine' del poema eroico: un problema di poetica cinquecentesca”. Per la ENERGEIA si veda Musacchio, "Lo stile del nuovo poema epico rinascimentale". 
tivi: "retto", "quasi retto", "obliquo" e "quasi obliquo". La "narrazione retta è quella per la quale il poeta racconta come da sé". L'esempio che Verdizzotti porta a proposito di quello che per Platone e per Aristotele rappresentava lo stile non 'drammatico', l'opposto insomma dello stile omerico, è preso proprio da Virgilio: "come fa Virgilio nel primo libro della sua Eneide, nel quarto, nel quinto, nel sesto" 58 . Verdizzotti implicitamente mostra che lo stile virgiliano non segue affatto il modello omerico. Il modo 'drammatico' ha la sua parte nell' Eneide, ma il modo 'narrativo puro e semplice' vi ha un ruolo rilevante. Verdizzotti era un grande ammiratore di Ariosto, e sebbene fosse un amico di Tasso confessava in privato che secondo lui il Furioso era l'esempio supremo ("tanto da me amato e con tutta l'anima mia onorato sempre") ${ }^{59}$, anche se sapeva bene che il gusto dei letterati contemporanei era diventato anti-ariostesco. E infatti, nel poema epico che stava scrivendo, aveva deciso di rispettare l'unità d'azione prescritta dagli aristotelici ("una sola azione contiene in se") E Verdizzotti chiarisce che la tecnica dello 'incastro narrativo' non è la stessa cosa che il modo 'mimetico', classificando quel tipo di narrazione come una narrazione "quasi retta", cioè "quella per la quale il poeta suol narrare ... ma introducendo qualche persona a raccontarla". E appunto alla 'narrazione quasi retta' assegnava il racconto di Enea nell' Eneide "in tutto il libro secondo et in tutto il terzo".

Raro è che i critici rinascimentali manifestino questa chiaroveggenza rispetto al problema della voce del narratore. Ancor più raramente tuttavia dimostrano di saper distinguere non solo l'autore materiale del poema dal suo specifico narratore ('l'autore implicito') 60 ma addirittura quest'ultimo dal personaggio del Narratore che è tradizionale nello stile volutamente orale dei Romanzi. Di questa crassa confusione l'esempio più insigne è forse quello di Castelvetro che sembra imputare ad Ariosto le opinioni espresse dal personaggio un po' fanfarone del Narratore messo in scena nel Furioso.

58 Verdizzotti, Breve discorso attorno alla narrazione poetica, 7.

${ }^{59}$ Da una lettera a Orazio Ariosto del 1585, in Verdizzotti, Lettere a Orazio Ariosti, 4.

${ }^{60}$ L'espressione 'autore implicito' è il termine accettato dai narratologhi ("l'autore come si rivela nell'opera" come è definito da Segre, "an official scribe" nella definizione di Booth); e che per convenienza possiamo chiamare Omero o Ariosto, pur ben tenendo presente che non bisogna confonderli con le persone anagrafiche degli autori ("it is essential not to confuse author and narrator", ricorda Chatman, Story and Discourse, 147). 
Ariosto (e Boiardo prima di lui) ha seguito la convenzione stabilita dai Cantari di esibire una fittizia oralità in un racconto immaginato come eseguito davanti ad un pubblico fisicamente presente e direttamente interpellato dal personaggio di un narratore ("Signori e buona gente che audire / séte venuti inanzi al mio cospetto / pregar ne vo' l'onnipotente sire / che m'ascoltiate in pace con diletto; / ed io vi conterò in questo dire / del valoroso re magno e perfetto") ${ }^{61}$.

Nel Furioso la figura del Narratore così come è tradizionalmente mantenuta nei Cantari, viene ripresa da Ariosto, ma sublimata e al tempo scardinata. Il narratore del Furioso non è il personaggio generico e incolore dei Cantari. Ha una sua distinta personalità. Si lascia andare a lunghi commenti e giudizi. Ma in effetti questi giudizi sono spesso sfasati rispetto alla storia, perché il Narratore si proietta, attraverso le sue proprie parole, come un individuo a cui il racconto che sta facendo risulta incomprensibile tenuto conto del suo limitatissimo punto di vista ${ }^{62}$.

${ }^{61}$ La Spagna, I, 3. Eventualmente il Narratore dichiara la sua intenzione di interrompere il racconto ("Nell'altro dir dirò la diceria / che 'I messagio a Marsilio racontava", La Spagna, I, 44) e di riprenderlo ("Signori, io dissi nel cantar primiero / sì come al re Marsilio era tornato", La Spagna, II, 2). Linterruzione e la ripresa possono essere ripetute con variazioni in ogni successivo canto. Per esempio nella lunghissima Spagna, il VI canto comincia "Voi buona gente, se volete odire: in pace tutti vi state a sedere" (La Spagna, VI, 2), e finisce "Signori, i' vo finir questo cantare/e gire a bere e rinfrescarmi alquanto" (La Spagna, VI, 46). A volte l'autore del Cantare finge di assegnare al Narratore un ruolo di semplice testimone o meglio di trascrittore di un racconto che è un evento storico, dunque assolutamente vero ("Raconta el libro", "il libro sì mi narra", La Spagna, II, 30). Si veda per esempio la dichiarazione del narratore di Li Fatti de Spagna. Non solo questa "veraxe istoria" è ripresa da Turpino ma addirittura è di ispirazione divina: "Una note el me vene uno angelo in vixione anonziare che io dovesse scrivere la dita istoria si voleva essere salvo" (Li Fatti di Spagna, I, 434). Il racconto dunque sarebbe stato dettato direttamente senza intervento del Narratore che dunque non viene presentato come un narratore vero e proprio ma piuttosto come un semplice scriba.

62"Ciò che è particolare nel Furioso è che il Narratore impersona in un racconto impregnato di profonda idealità una mentalità prosaica e ottusamente (ma anche astutamente) realistica. È un borghese calato in un mondo aristocratico, il quale senza fini dichiaratamente dimistificatori commenta a volte in modo impertinente, sempre comunque in maniera poco pertinente, ciò che succede, secondo la sua limitata ottica. Sono commenti che tendono a generalizzare e a moralizzare sulla base di quanto effettivamente sta avvenendo nella storia ma interpretandolo in modo sfasato, anzi a volte grottescamento erroneo, e che ci permet- 
Una volta che ha introdotto, interrotto e ripreso il suo racconto, il Narratore del Furioso (come nei Cantari) ha concluso le sue funzioni: il suo ruolo termina qui. E la storia viene raccontata indipendentemente dal suo punto di vista e non narrata dalla sua voce; il Narratore ufficiale non è insomma in alcun modo "il personaggio il cui punto di vista orienta la prospettiva narrativa" 63 .I propositi di questo personaggio (che si dichiara il narratore della storia ma non lo è effettivamente) rimangono semplicemente i suoi giudizi (individuali e legati alle limitazioni della sua personalità), non colorano in alcun modo la narrazione. La 'prospettiva narrativa' è quella fornita dall'effettivo narratore, il cosidetto 'narratore implicito'. Questo vero narratore del Furioso si esprime attraverso un sistema multiforme e polivalente di 'voci', più o meno 'distanti' dalla storia narrata, presenti nei vari episodi in modo ogni volta differente, in un ventaglio di modi narrativi che vanno da un distacco ironico e divertito fino a una intensa partecipazione ${ }^{64}$.

tono in tal modo di mettere a fuoco, per effetto del contrasto, il significato dell'episodio, in quell'intercalare fra una profondamente sofferta passionalità e un più superficiale, per quanto più appariscente, scetticismo, che è il tono tipico del Furioso" (Musacchio, Amore, Ragione e Follia, 29-30). La possibilità lettera-ria di un narratore incapace di comprendere la storia che egli stesso sta raccontando, naturalmente, verrà impiegata in certi esempi di romanzo moderno ("le romancier ... fera raconter l'histoire par un narrateur auquel le lecteur ne peut pas se fier entièrement soit parce qu'il veut nous tromper ou qu'il manque de lucidité", Bourneuf e Ouellet, L'Univers du Roman, 87). Non è il caso tuttavia del Furioso in cui il personaggio del Narratore non è il vero narratore della storia. Solo i suoi giudizi e commenti sono dunque sfasati ('mancano di lucidià', nella terminologia di Bourneuf e Ouellet).

${ }^{63}$ Segre, "Testo letterario, interpretazione, storia," 36-37. Gli sviluppi della discussione contemporanea sul 'Punto di Vista' (o sulla 'Voce') sono descritti in Friedman, "Point of View in Fiction"; e in Booth, "Distance and Point-ofView"; ma si vedano le sempre acute pagine di Genette nel Discours du récit.

${ }^{64} \mathrm{Si}$ veda il commento del Narratore cinicamente incredulo sulla presunta verginità di Angelica ("Forse era ver, ma non però credibile / a chi del senso suo fosse signore", I, 56), totalmente inappropriato nel contesto del tema principale dei due Orlandi, in cui tale verginità è il motore principale per l'azione; e come contrasto l'altro commento del Narratore, sulla sofferenza di Orlando quando scopre di aver perduto Angelica, momento culminante dell'opera ("Credere a chi n'ha fatto esperimento / che questo è 'I duol che tutti gli altri passa", XXIII, 112), commento improntato alla simpatia e alla partecipazione emotiva. A proposito della partecipazione del narratore alla follia di Orlando, intensamente sofferta e al tempo stesso estremamente sobria e contenuta, si potrebbe osservare che 
Il divario tra l'interpretazione della storia, quale è dichiarata dai commenti del personaggio del Narratore, e il senso del racconto, quale si sprigiona dall'opera per noi lettori, è tale e così palese che è difficile comprendere come Castelvetro, per esempio, abbia potuto attribuire all'autore del Furioso i limitati propositi moraleggianti del personaggio del Narratore. Eppure questa assurda identificazione risulta evidente dalle sue parole quando si indigna perché Ariosto ha tralasciato "l'ufficio di narratore che era suo proprio" e ha assunto quello di "predicatore et di correggitore de" costumi". Ė ovvio in tali parole di Castelvetro che questo rimprovero è rivolto non al personaggio del Narratore, ma all'autore del poema identificato grossolanamente con il primo. I propositi dichiarati dal personaggio del Narratore, in generale all'inizio di ogni canto, sarebbero dunque l'espressione del narratore stesso, della 'voce narrativa' del Furioso, anzi addirittura di Ariosto.

Soltanto una fanatica insistenza a voler condannare la letteratura dei Romanzi, e in particolare il più illustre fra i suoi autori, può spiegare una così sconcertante mancanza di acume critico. Va osservato che dei vari punti chiave sollevati dalla lettura della poetica aristotelica circa la struttura del poema eroico, quella riguardante la voce del narratore è la questione che meno universalmente sembra aver eccitato lo spirito critico dei vari partecipanti al dibattito sviluppatosi nel corso del " 500 . Alcuni dei più autorevoli non ne hanno affatto parlato. Trissino è forse il piu rimarchevole tra di essi, tenuto conto della sua proclamata aderenza ai precetti di Aristotele e della sua altrettanto proclamata ammirazione per Omero e il suo stile. In effetti se avesse voluto sposare l'opinione che il poeta deve parlare il meno possibile, gli si presentava la possibilità di sottolineare mediante quella frase di Aristotele una ulteriore ragione per il suo giudizio negativo su Virgilio e ancor più sui suoi imitatori rinascimentali, e per il suo disprezzo per i Romanzi e specificamente per Ariosto65. Tra Giraldi e Bernardo Tasso intercorre un'interessante discussione teorica per via epistolare tra il 1556 e il 1559; si tratta di uno scambio in cui i problemi del poema epico vengono ampiamente trattati; ma il problema dell'intervento

osservare che Ariosto sembra adeguarsi alla caratterizzazione degli interventi d'autore preconizzati da Sartre (nella sua feroce polemica contro Mauriac): "il ne s'agit pas de définir, encore moins d'expliquer ... mais seulement de présenter des passions et des actes imprévisibles", Sartre, "M. François Mauriac et la liberté" I, 19. Distacco che naturalmente è solo apparente. Per questa varietà di voci narrative, l'Orlando Furioso si apparenta forse al metodo omerico più che all'uniformità della voce virgiliana.

${ }^{65}$ Si veda Musacchio. "Il poema epico ad una svolta". 
del poeta non viene affrontato ${ }^{66}$. Come si è visto Giraldi nel suo trattato ne accenna, ma solo tangenzialmente. Questi autori erano tutti anche praticanti dell'arte e non solo critici: Trissino con la sua Italia Liberata, Bernardo Tasso con il suo Amadigi, Giraldi con il suo Ercole. Potrebbe essere questa loro conoscenza pratica dei problemi della scrittura la ragione che li rendeva cauti nel pronunciarsi dogmaticamente e genericamente contro gli interventi del narratore?

La questione della voce del narratore sta ovviamente nel cuore stesso del processo creativo e gli autori ne erano inevitabilmente consci, mentre per i semplici critici, incapaci di vederne la rilevanza, il problema rimaneva astrattamente quello di una maggiore aderenza al nuovo manifesto letterario rappresentato dalla Poetica aristotelica. La distinzione tra i due modi del racconto messa in evidenza da Platone, doveva risultare opaca a questi ammiratori di un classicismo rappresentato essenzialmente dall'opera virgiliana, i quali si mostravano incapaci di leggere ed apprezzare la peculiarità dello stile omerico. La risposta dei lettori rinascimentali italiani di Omero infatti è lungi dall'essere entusiasta. Vi si trova sopratutto il rispetto per gli autori latini che veneravano Omero, più che l'ammirazione per Omero stesso. Si vedano per esempio le pagine di Giraldi Cinzio dove si spiega che Omero appariva grande nei tempi quando ancora non si era "veduto la forma del poeteggiare che si vide poscia al tempo di Vergilio". È chiaro che Giraldi non è in grado di scoprire uno stile omerico specifico ma vede solo un tentativo di scrittura che non ha ancora saputo trovare se stessa $^{67}$. Ma, mentre i critici potevano permettersi, come Castelvetro, il lusso di un'accettazione indiscriminata dei giudizi aristotelici, quelli fra questi critici che si erano cimentati nella scrittura di un proprio poema eroico si trovavano necessariamente davanti alla difficoltà di elaborare una specifica voce narrativa in ognuna delle loro opere. E non potevano pertanto offrire le medesime semplicistiche risposte.

Ne è esempio Tasso, il quale, dipartendosi dalla scelta del narratore taciturno e volutamente invisibile del Rinaldo, al momento di comporre la Gerusalemme Liberata, alcuni anni dopo, opterà per un narratore ancora più incline a emettere giudizi morali (in effetti scopertamente e irritabilmente bigotto a volte) di quanto non lo fosse stato il personaggio del Narratore del Furioso, tanto criticato da Castelvetro. Non solo, ma Tasso inventa una voce narrante dotata di una straordinaria ambiguità verso i propri personaggi, in una mescolanza di amore e di odio, di attrazione e di

${ }^{66}$ Giraldi Cinzio, Carteggio, 269-357.

${ }^{67}$ Giraldi Cinzio, Discorso dei romanzi, 67-68. 
ripugnanza che potrà addirittura sfociare in uno strano e affascinante rapporto di dominanza e di dipendenza del narratore rispetto ai personaggi da lui stesso creati68.

\section{UNIVERSITY OF ALBERTA}

\section{OPERE CITATE}

Aristotele. Poetics. Introduction, commentary and appendixes. A cura di Donald William Lucas. Oxford: Clarendon Press, 1968.

Aristotele. Poetica. A cura di Augusto Rostagni. Torino: Chiantore, 1945.

Aristotele. De arte poetica. Traduzione di William de Moerbeck. A cura di Lorenzo Minio-Paluello. Leiden: Brill, 1968.

Averroé. Commento al 'Perì Poietikês'. A cura di Carmela Baffioni. Milano: Coliseum Editore, 1990.

Barthes, Roland. "Introduction à l'analyse structurale des récits". Communications 8 (1966).

Benedetti, Laura. La sconfitta di Diana. Ravenna: Longo, 1996.

Booth. Wayne C. The Rhetoric of Fiction. Chicago: University of Chicago Press, 1961.

. "Distance and Point-of-View: an essay in classification". Essays in Criticism 11 (1961). Ristampa in Philip Stevick. The Theory of the Novel. New York: Free Press, 1967, pp. 87-107.

Bourneuf, Roland e Réal Ouellet. L'Univers du Roman. Paris: PUF, 1981.

Caburacci da Imola, Francesco. Trattato dove si dimostra il vero et novo modo di fare le imprese, con un breve discorso in difesa dell'Orlando Furioso di M. Lodovico Ariosto. Bologna: Gio. Rossi, 1580.

Castelvetro, Ludovico. Poetica d'Aristotele vulgarizzata et sposta. Vienna: Steinhofer, 1570

Chatman, Seymour. Story and Discourse. Ithaca: Cornell University Press, 1978.

Della Volpe, Galvano. Poetica del Cinquecento. Bari: Laterza, 1954.

Dolce, Ludovico. Lettera a Gabriele Giolito in Ludovico Ariosto, Orlando Furioso. Venezia: Giolito, 1544.

Else, Gerald F. Aristotle's Poetics: The Argument. Cambridge: Harvard Univeristy Press, 1957.

Friedman, Norman. "Point of View in Fiction: The Development of a Critical Concept". PMLA 70 (1965): 1160-1184.

Fornari, Simon. Apologia brieve sopra tutto l'Orlando Furioso in La Spositione di M.

${ }^{68}$ Musacchio, "Degne di un chiaro sol, degne d'un pieno teatro - Private heroism and public glory for the knights of Italian Renaissance Chivalry Romances". Più generalmente sull'ambiguità dell'atteggiamento di Tasso riguardo al significato stesso del propriproprio poema si veda Benedetti, La sconfitta di Diana. 
Simon Fornari da Rheggio sopra l'Orlando Furioso di M. Ludovico Ariosto. Firenze: Lorenzo Torrentino, 1549.

Galilei, Galileo. Considerazioni al Tasso in Galileo Galilei, Scritti Letterari. A cura di Alberto Chiari. Firenze: Le Monnier, 1943.

Genette, Gérard. Discours du récit in Genette, Figures III. Paris: Seuil, 1972, pp. 65-278.

Giraldi Cinzio, Giovambattista. Discorso dei romanzi. A cura di Laura Benedetti, Giuseppe Monorchio, Enrico Musacchio. Bologna: Millennium, 1999.

—. Carteggio. A cura di Susanna Villari. Messina: Sicania, 1996.

Halliwell, Stephen. Aristotle's Poetics. Chapel Hill: University of North Carolina Press, 1986.

Javitch, Daniel. Proclaiming a Classic. The Canonization of Orlando Furioso. Princeton: Princeton University Press, 1991.

La Spagna, Poema cavalleresco del secolo XIV. A cura di Michele Catalano. 3 voll. Bologna: Commissione per i testi di lingua, 1939.

Li Fatti de Spagna in Poemi cavallereschi del Trecento. A cura di Giuseppe Guida Ferrero. Torino: UTET, 1965.

Lubbock, Percy. The Craft of Fiction. Londra: Jonathan Cape, 1921.

Maggi, Vincenzo e Bartolomeo Lombardi. In Aristotelis librum de poetica communes explanationes. Venezia: Valgrisi, 1550.

Malatesta, Gioseppe. Della nuova poesia, overo della difesa del Furioso dialogo. Verona: Sebastiano dalle Donne, 1589.

Mazzoni, Jacopo. Introduzione alla difesa della 'Commedia' di Dante. A cura di Enrico Musacchio e Gigino Pellegrini. Bologna: Cappelli, 1982.

Morsolin, Bernardo. Giangiorgio Trissino. Vicenza: Burato, 1878.

Musacchio, Enrico. Amore, Ragione e Follia. Una rilettura dell'Orlando Furioso. Roma: Bulzoni, 1983.

"Degne di un chiaro sol, degne d'un pieno teatro - Private heroism and public glory for the knights of Italian Renaissance Chivalry Romances". In $Z$ zagadnien literatury, kultury i jezyka. Studia ofiarowane Professorowi Edwardowi Mozejce. A cura di Bozeny Tokarz. Katowice: 2002. 75-83.

_. "Il poema epico ad una svolta: Trissino tra modello omerico e modello virgiliano”. Italica 80:3 (2003): 334-352.

—. "Il 'principio, mezzo e fine' del poema eroico: un problema di poetica cinquecentesca”. Quaderni d'Italianistica 20:2 (2004): 21-43.

- "Lo stile del nuovo poema epico rinascimentale". Letteratura italiana antica 6 (2005): 369-389.

Omero. Iliade. A cura di Rosa Calzecchi Onesti. Torino: Einaudi, 1977.

Patrizi da Cherso, Francesco. Della poetica. A cura di Danilo Aguzzi Barbagli.

Firenze: Istituto Nazionale di Studi sul Rinascimento, 1970.

Piccolomini, Alessandro. Annotationi di M. Alessandro Piccolomini nel libro della Poetica d'Aristotele. Venezia: Giovanni Guarisco, 1575.

Pigna, Giovan Battista. I Romanzi. Venezia: Valgrisi, 1554.

Pirotti, Umberto. "Benedetto Varchi e l'aristotelismo del Rinascimento". Convivium 31 (1963): 280-311. 
Platone. La Repubblica. Testo e traduzione a cura di Francesco Adorno e Francesco Gabrieli. Milano: BUR, 2004.

Robortello, Francesco. In librum Aristotelis de arte poetica explicationes. Firenze: Torrentini, 1548 (riproduzione Monaco: Fink Verlag, 1968).

Sartre, Jean-Paul. "M. François Mauriac et la liberté". Situations, I, Paris, Gallimard, 1947, pp. 33-52.

Segni, Agnolo. Lezioni intorno alla poesia (1573) in Trattati di Poetica e Retorica del Cinquecento, A cura di Bernard Weinberg. Bari: Laterza, 1972, vol. 3.

Segre, Cesare. "Testo Letterario, interpretazione, storia: linee concettuali e categorie critiche". In Letteratura Italiana, vol. 4, L'interpretazione. Torino: Einaudi, 1985.

Sigonio, Carlo. Emendationum (Liber II, cap. XXXIII) in Opera Omnia. T. 6. Milano: P. Argelatus Bononiensis, 1737.

Speroni, Sperone. Epistolario in Speroni, Opere, vol. 5. Venezia, Domenico Occhi, 1740.

- Dialogo primo sopra Virgilio in Speroni, Opere, vol. 2. Venezia, Domenico Occhi, 1740.

- Discorso quinto sopra Virgilio in Speroni, Opere, vol 2. Venezia, Domenico Occhi, 1740 .

Tasso, Torquato. Rinaldo. A cura di Luigi Bonfigli. Bari: Laterza, 1936.

- Discorsi dell'arte poetica e del poema eroico. A cura di Luigi Poma. Bari: Laterza, 1964.

—. Lettere poetiche. A cura di Carla Molinari. Parma: Guanda, 1995.

Tiraboschi, Girolamo. Storia della letteratura italiana. Roma: Salvioni, 1785.

Trissino, G.G. La Poetica (La Quinta Divisione, e La Sesta Divisione) in Trattati di Poetica e Retorica del Cinquecento, vol. 2. A cura di Bernard Weinberg. Bari: Laterza, 1970.

Varchi, Benedetto. Opere. Trieste: Lloyd Austriaco, 1859.

Verdizzotti, Mario. Lettere a Orazio Ariosti. Bologna: Scelta di Curiosità Letterarie, Commissione per i testi di Lingua, 1969.

- Breve discorso attorno alla narrazione poetica (1588) in Trattati di Poetica e Retorica del Cinquecento. A cura di Bernard Weinberg. Vol. 4. Bari: Laterza, 1974.

Vettori, Pietro. Commentarii in primum librum Aristotelis de arte poetarum, Firenze: Giunta, 1560 (riproduzione Monaco: Fink Verlag, 1967).

Weinberg, Bernard. A History of Literary Criticism in the Italian Renaissance. Chicago: University of Chicago Press, 1961. 